Yukio MARUYAMA ${ }^{1}$, Yoshifumi KAWAMURA 1 , Takao ISOGAI2 ${ }^{2}$ Nobuo NOMURA ${ }^{3}$ and Naoki GOSHIMA ${ }^{3}$

1 Japan Biological Informatics Consortium, JAPAN

2 Graduate School of Pharmaceutical Sciences, The University of Tokyo, JAPAN

${ }^{3}$ National Institute of Advanced Industrial Science and Technology, JAPAN

\title{
ABSTRACT
}

HGPD (http://www.HGPD.jp/) is a unique database that stores information on a set of human Gateway entry clones in addition to protein expression data. HGPD was launched in November 2008 and, since then, 33,275 human Gateway entry clones have been constructed from ORFs of full-length cDNAs, thus representing the largest collection in the world. Sequence information and protein expression of Gateway entry clones can also be retrieved from HGPD. The majority of analysis data for CDNA sequences in HGPD are shared with the FLJ Human cDNA Database (http://flj.hinv.jp/v01/cgi/index.cgi), which was constructed as human cDNA sequence analysis database focusing on mRNA varieties resulting from variations in transcription start site (TSS) and splicing.

\section{Data SET}

- Approximately 43,000 kinds of Gateway entry clone are constructed (see following table). This construction is covered above $70 \%$ of human coding protein.

(Identified 2010/10/10)

\begin{tabular}{l|c}
\hline \multicolumn{1}{c|}{ Type of Gateway Entry Clones } & Numbers \\
\hline $\mathrm{N}$ (stop codon type of C-terminal) & 17,802 \\
\hline F (fusion type of C-terminal) & 25,447 \\
\hline
\end{tabular}

- This human Gateway entry clone resource is named HUPEX (Human Proteome Expression-resource).

\section{WEB INTERFACE}

- ID search (DDBJ / EMBL / GenBank Accession, Gene symbol, etc.)

- Keyword search
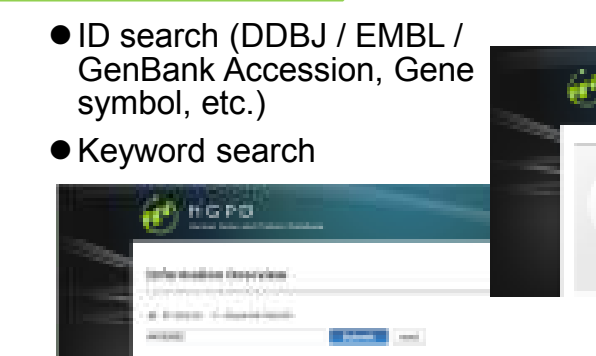
(http://www.HGPD.jp/) is наров Top View

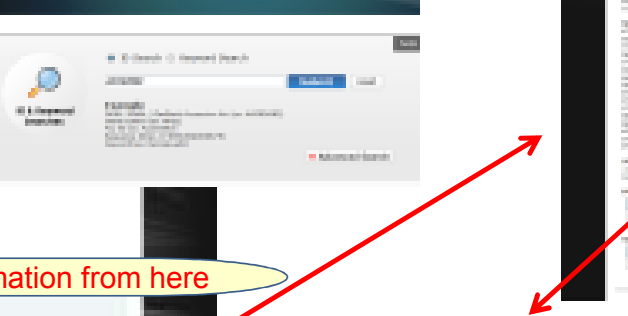

Information Overview: Link to other information from here

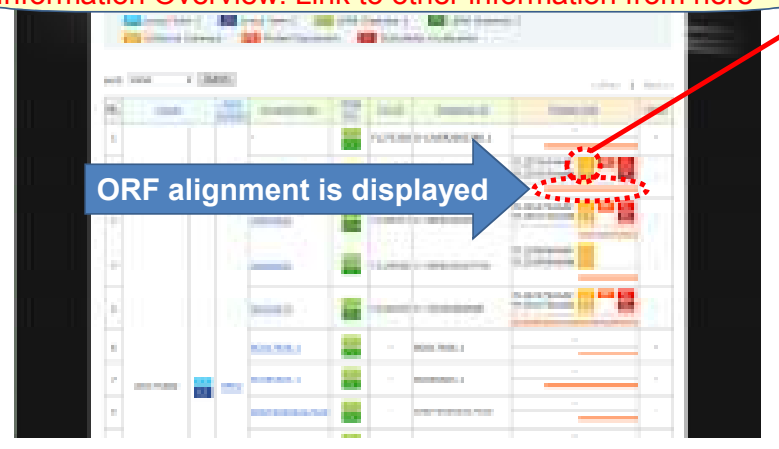

\section{HOW TO GET HUPEX}

- HUPEX is distributed from NITE Biological Resource Center (NBRC) http://www.nbrc.nite.go.jp/e/hgentry-e.html (*1).

\section{FUTURE WORKS}

- HUPEX is categorized by protein function (protein kinase, protein phosphatase, transcription factor, GPCR, etc.).

- Linkage to ESPRESSO (http://mbs.cbrc.jp/ESPRESSO/) $\left({ }^{*} 2\right)$ which is a sequence-based predictor for estimating protein expression and solubility is added.

- Subcellular localization image data of proteins with fluorescent proteins at the $\mathrm{N}$ - or $\mathrm{C}$-terminal which are expressed in HeLa cells by using Gateway entry clones (*3) will be launched.
- 17,821 kinds of human proteins with a C-terminal V5 and His tag are expressed using SDS-PAGE.

- Sequence information of Gateway entry clone can be get from download site. The latest version is 2.0 (identified 2010/10/10).

(http://riodb.ibase.aist.go.jp/hgpd/sys_info/download.html)

- Human protein expression data (SDS-PAGE) can be get from "Protein Expression" view of each Gateway entry clone. 